Portland State University

PDXScholar

\title{
An Essay on Proof, Conviction, and Explanation: Multiple Representation Systems in Combinatorics
}

\author{
Elise Nicole Lockwood \\ Oregon State University \\ John Caughman \\ Portland State University, caughman@pdx.edu \\ Keith Weber \\ Rutgers University - New Brunswick/Piscataway
}

Follow this and additional works at: https://pdxscholar.library.pdx.edu/mth_fac

Part of the Applied Mathematics Commons

Let us know how access to this document benefits you.

\section{Citation Details}

Lockwood, E., Caughman, J. S., \& Weber, K. (2020). An essay on proof, conviction, and explanation: multiple representation systems in combinatorics. Educational Studies in Mathematics, 103(2), 173-189.

This Post-Print is brought to you for free and open access. It has been accepted for inclusion in Mathematics and Statistics Faculty Publications and Presentations by an authorized administrator of PDXScholar. Please contact us if we can make this document more accessible: pdxscholar@pdx.edu. 


\section{An essay on proof, conviction, and explanation: Multiple representation systems in combinatorics}

There is a longstanding conversation in the mathematics education literature about proofs that explain versus proofs that only convince. In this essay, we offer a characterization of explanatory proofs with three goals in mind. We first propose a theory of explanatory proofs for mathematics education in terms of the representation systems. Then, we illustrate these ideas in terms of combinatorial proofs, focusing on binomial identities. Finally, we leverage our theory to explain audience-dependent and audience-invariant aspects of explanatory proof. Throughout, we use the context of combinatorics to emphasize points and to offer examples of proofs that can be explanatory or only convincing, depending on how one understands the claim being made.

Keywords: Proof, Proofs that explain and convince, Combinatorics, Binomial identities

Elise Lockwood

Oregon State University

Elise.Lockwood@oregonstate.edu

(541) 737-5164

John S. Caughman

Portland State University

Keith Weber

Rutgers University 


\section{Introduction and Motivation}

Consider the following identity. For all nonnegative integers $m \leq k \leq n$, the binomial coefficients satisfy:

$$
\left(\begin{array}{l}
n \\
k
\end{array}\right)\left(\begin{array}{c}
k \\
m
\end{array}\right)=\left(\begin{array}{c}
n \\
m
\end{array}\right)\left(\begin{array}{l}
n-m \\
k-m
\end{array}\right)
$$

Which of the proofs below do you consider to be explanatory?

Proof 1. We show both sides of (1) count the number of $k$-person committees with an $m$-person subcommittee, all chosen from a set of $n$ people.

On the left, we first select a $k$-person committee (there are $\left(\begin{array}{l}n \\ k\end{array}\right)$ ways to do so), and then select an $m$-person subcommittee from the $k$-person committee (there are $\left(\begin{array}{l}k \\ m\end{array}\right)$ ways to do so).

On the right, we first select an $m$-person subcommittee (there are $\left(\begin{array}{l}n \\ m\end{array}\right)$ ways to do so), and then select the remaining $k-m$ people on the committee from the remaining $n-m$ people (there are $\left(\begin{array}{c}n-m \\ k-m\end{array}\right)$ ways to do so).

Because both sides of (1) each enumerate the same set of objects, equality holds.

Figure 1. An enumerative proof of (1).

Proof 2. Using the identity $\left(\begin{array}{l}n \\ k\end{array}\right)=\frac{n !}{(n-k) ! k !}$, we use algebra to deduce (1) as follows:

$$
\begin{aligned}
& \left(\begin{array}{l}
n \\
m
\end{array}\right)\left(\begin{array}{c}
n-m \\
k-m
\end{array}\right)=\left(\frac{n !}{(n-m) ! m !}\right)\left(\frac{(n-m) !}{((n-m)-(k-m)) !(k-m) !}\right) \\
& =\quad\left(\frac{n !}{m !(n-k) !(k-m) !}\right)\left(\frac{k !}{k !}\right) \\
& =\quad\left(\frac{n !}{(n-k) ! k !}\right)\left(\frac{k !}{(k-m) ! m !}\right) \\
& =\quad\left(\begin{array}{l}
n \\
k
\end{array}\right)\left(\begin{array}{l}
k \\
m
\end{array}\right)
\end{aligned}
$$

Figure 2. An algebraic proof of (1).

In our experience, many mathematics educators tend to find Proof 1 to be explanatory, while few find Proof 2 to be explanatory. In what follows, we provide a theory of proof explanation in which either proof can be judged as explanatory, depending upon the motivation and background knowledge of the reader. This theory also offers a means to explain why Proof 1 may be more likely to be judged as explanatory than Proof 2.

Our main goal is to provide a theoretical characterization of explanatory proof, particularly leveraging examples of proofs in combinatorics to do so. The purpose of this paper is threefold. First, we propose a theory of explanatory proofs for mathematics education in terms of the representation systems in which proofs are developed, written, and understood. Mathematics educators have long contended that there is an important distinction between proofs that explain and proofs that only convince (e.g., Hanna, 1990; Hersh, 1993; Pulte, Hanna, \& Jahnke, 2009; Stylianides, Sandefur, \& Watson, 2016; Weber, 2010a). However, the community also acknowledges that there is no consensus on what an explanatory proof in mathematics education is or should be; indeed, many believe this concept is poorly understood (Bartlo, 2013; Stylianides et al., 2016; Raman, 2003). Consequently, a theory of explanatory proof, adequate for the aims of mathematics educators, is urgently needed. We provide a candidate for such a theory. Second, 
we illustrate why the field of combinatorics in general, and the topic of binomial identities in particular, provides a fruitful context to study proofs that convince and explain. Third, we seek to leverage our theory to explain audience-dependent and audience-invariant aspects of explanatory proof. Specifically, we maintain that our theory can elucidate why the explanatory nature (or lack thereof) of some proofs will depend both on who is reading the proof and what is to be explained, while the explanatory nature of other proofs will be agreed upon by most students and mathematicians.

\section{Background Literature and Relevant Theoretical Perspectives}

\section{What Is a Proof?}

We draw on a definition of proof (Figure 3) that Stylianides (2007) has developed for characterizing proof in school mathematics.

Proof is a mathematical argument, a connected sequence of assertions for or against a mathematical claim, with the following characteristics: 1 . It uses statements accepted by the classroom community (set of accepted statements) that are true and available without further justification; 2. It employs forms of reasoning (modes of argumentation) that are valid and known to, or within the conceptual reach of, the classroom community; and 3. It is communicated with forms of expression (modes of argument representation) that are appropriate and known to, or within the conceptual reach of, the classroom community (Stylianides, 2007, p. 291).

Figure 3. Stylianides' (2007) definition of proof.

We add several clarifications to Stylianides' (2007) characterization of proof. First, proofs form a subset of mathematical arguments; every proof is a mathematical argument but not every mathematical argument is a proof. Second, Stylianides' aim was to define proof in a way that respected the role of the classroom community while retaining the mathematical integrity of proof (e.g., the modes of argumentation are valid). Here, related to Stylianides' characterization, we interpret a true statement as one the professional mathematical community accepts as true, an inferential scheme as valid if that community would find it valid, and a representation as appropriate if that community would find it suitable for proof. In this sense, being a proof must satisfy constraints apart from any individual or classroom community (Balacheff, 2009). Third, when an individual decides if an argument is a proof, she must estimate the community, and specifically, the cognition of professional mathematicians. For instance, for a particular inference to be allowable in a proof, it is not enough for the individual to accept it; she must also believe it to be valid for the community. Hence, deciding whether an argument is a proof involves a social judgment. We will later contrast this with deciding whether an argument is convincing or explanatory, which we will frame as psychological judgments. Fourth, by proving, we are referring to the activity in which an individual is trying to construct an argument that conforms to the norms in Stylianides' characterization.

\section{The Value of Explanatory Proofs}

Mathematics educators have recommended that proof play a central role in mathematics classrooms (see Stylianides et al., 2016, for a summary). One line of argument is that a proof demonstrates that a theorem is a logically necessary consequence of statements that are accepted 
as true; accordingly, a proof can provide students with psychological certainty that theorems are true (Harel \& Sowder, 1998). However, many scholars have argued that a proof can explain why a theorem is true to students (deVilliers, 1990; Hanna, 1990; Hersh, 1993; Weber, 2010a), noting that proofs often play an explanatory role in professional mathematicians' practice (e.g., deVilliers, 1990; Steiner, 1978).

To highlight this distinction, researchers have distinguished between proofs that (only) convince and proofs that simultaneously convince and explain. We refer to the latter as explanatory proofs. Hanna (1990) makes the distinction as follows: a proof that proves "shows only that a theorem is true; it provides evidential reasons alone" (p.10), while an explanatory proof "also shows why a theorem is true; it provides a set of reasons that derive from the phenomenon itself" (p.10). We thus interpret Hanna's term "proofs that prove" as being synonymous with proofs that only convince. Hanna urges mathematics educators to consider the pedagogical value of explanatory proofs. Hersh (1993) agrees, arguing that although the primary purpose for proofs in mathematical practice is to provide conviction, the primary purpose of proof in classrooms should be to provide explanation: "In mathematical research, the purpose of proof is to convince. The test of whether something is a proof is whether it convinces qualified judges. In the classroom, on the other hand, the purpose of proof is to explain" (p.389). Numerous mathematics educators endorse the conclusion that explanatory proofs should play an important role in mathematics classrooms (e.g., deVilliers, 1990; Stylianides et al., 2016; Weber, 2010a).

\section{What Is an Explanatory Proof in Mathematics Education?}

If we accept that explanatory proofs should play a role in classrooms, then it is important to have a good sense of what an explanatory proof is. However, mathematics educators have observed that no widely shared operationalization of explanatory proof exists (Bartlo, 2013; Raman, 2003; Weber, 2010a). Indeed, we find it telling that in our reading of Pulte et al.'s (2009) edited volume on proof and explanation, we found no operationalized definition that would distinguish proofs that explain from proofs that merely convince.

Following Bartlo's (2013) literature review, we note that mathematics educators' attempts to clarify the meaning of explanatory proofs typically take one of three forms. First, mathematics educators have adapted definitions of explanatory proof from the philosophy literature. For instance, Steiner (1978) states that a proof is explanatory when it reveals a 'characteristic property,' where a property is characteristic if the proof would fail if the property was false and one could use the same proof technique but substitute another characteristic property to prove a new theorem. Hanna (1990) defines explanatory proofs in terms of characteristic properties: "Following Steiner (1978), I will say a proof explains when it shows what "characteristic property' entails the theorem that it purports to prove" (p.10). However, such definitions evaluate the explanatory nature of a proof independently of the student reading the proof (Bartlo, 2013; Weber, 2010a). If one accepts the premise that a proof that is explanatory to a mathematician might not be explanatory to a student, then objective, audience-independent definitions will be incomplete for mathematics educators' purposes.

Second, mathematics educators have provided examples of proofs that are (or are not) explanatory. Bartlo (2013) summarized the typical examples:

These often involve showing proofs that involve pictures and stating that the visual proofs explain what they are proving, and showing proofs by induction or involving complicated 
algebra and stating that those proofs only show that a statement is true without offering any explanation (Bartlo, 2013, p.34).

For instance, Hanna (1990) offers several proofs for a sum of the first $n$ positive integers. She exemplifies a proof that proves with mathematical induction in Figure 4, saying:

Now, this is certainly an acceptable proof: it demonstrates that a mathematical statement is true. What it does not do, however, is show why the sum of the first $n$ integers is $\frac{n(n+1)}{2}$ or what characteristic property of the sum of the first $n$-integers might be responsible for the value $\frac{n(n+1)}{2}$ (Hanna, 1990, p.10).

Hanna presents the diagrammatic argument in Figure 5 as a proof that explains (although she does not specify what characteristic property this proof relies on or what is being explained).

\section{A proof that proves}

Proof by mathematical induction:

For $n=1$ the theorem is true.

Assume it is true for an arbitrary $k$.

Then consider:
$S(k+1)=S(k)+(k+1)=\frac{n(n+1)}{2}+(n+1)=\frac{(n+1)(n+2)}{2}$

Therefore the statement is true for $k+l$ if it is true for $k$.

By the induction theorem, the statement is true for all $n$.

Figure 4. A proof that proves (Hanna, 1990).

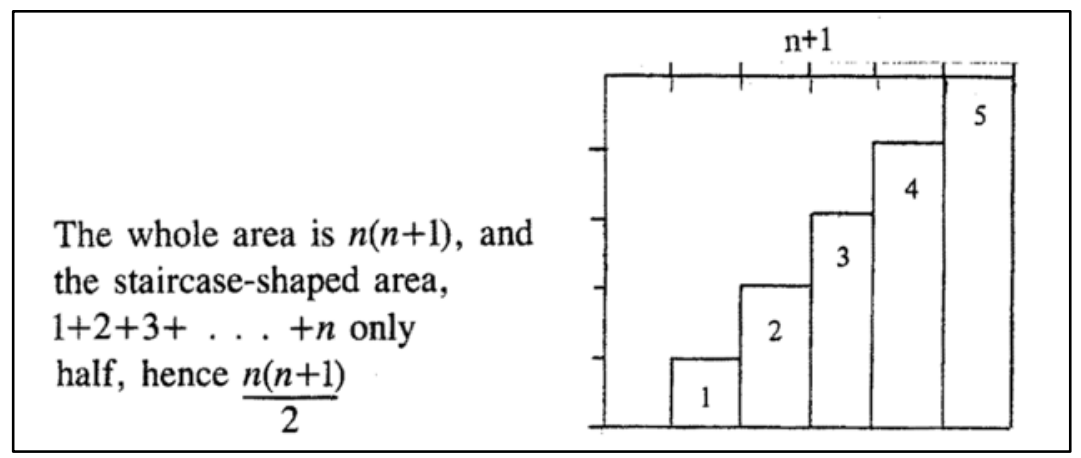

Figure 5. A proof that explains (Hanna, 1990).

These examples help frame debates on explanatory proofs but have not led to consensus on what makes a proof explanatory. Inductive proofs are often considered paradigmatic cases of proofs that are not explanatory (e.g., Lange, 2009), yet some mathematics educators have argued that such proofs can be explanatory (e.g., Harel, 2001; Stylianides, et al., 2016).

The third approach for defining explanatory proofs posits that the explanation from a proof is rooted in students' proving activity (Balacheff, 2009; Nunokawa, 2009; Raman, 2003; Stylianides, et al., 2016; Weber, 2010a; Weber \& Alcock, 2004). A commonality in these approaches is that in the process of proving, one may engage in argumentative processes that provide insight as to why a theorem is true, but that may be insufficiently rigorous to qualify as a 
proof. If the proof itself closely resembles the informal reasoning processes used to create it, then it qualifies as explanatory. This reminds us of Garuti et al.'s (1998) construct of cognitive unity, in which cognitive unity is achieved whenever an individual's process used to form or evaluate a conjecture is related to the proof they constructed to establish the conjecture. If cognitive unity is achieved, the (possibly non-rigorous) insights that explain why a theorem is true are present to the individual in the proof that she produces, so the proof is viewed as explanatory to the individual. While this approach is useful for illuminating how explanation and proof may coevolve in the proving process (Nunokawa, 2009), it is limited in the following respect: If explanation is by definition rooted in proving activity (Balacheff, 2009; Nunokawa, 2009), then it would seem that all proofs, if properly understood, are explanations. Balacheff (2009) explicitly endorses this position. However, this is in contrast with the claims of Hanna (1990) and Hersh (1993) that some proofs are not explanatory and thus undesirable to use in lectures. We also agree with Bartlo (2013) that it seems possible for a proof to be explanatory to an individual without the individual linking the proof to some other activity or argument. For instance, we believe most combinatorialists would find Proof 1 to be explanatory when they read it, without reflecting on how the proof might have been generated.

We agree with Bartlo (2013) that each of the three approaches above did not provide ${ }^{1}$ an adequate characterization of explanatory proof for mathematics educators' purposes, but we also believe that they provide some insights into the nature of explanatory proof for which an adequate theory should account. To summarize:

- Whether a proof is explanatory should, in some cases, depend upon who is reading the proof.

- Nonetheless, there is some uniformity in mathematics educators' judgments on whether some proofs are explanatory.

- Proofs that explicitly rely on visual reasoning are frequently judged as explanatory.

- Purely algebraic demonstrations are often not considered explanatory, although they can sometimes be explanatory to some audiences.

- Individuals' engagement in the proving process, especially if they can relate the process used to understand why a theorem is true to the resulting proof, is important for determining whether a proof is explanatory.

We now present a theory that we contend accounts for each of these phenomena.

\section{Characterizing Proof, Conviction, and Explanation in Terms of Representation Systems}

\section{Representation Systems}

Recall that in Stylianides' characterization of proof, an argument must be presented in a mode of representation that is acceptable to the appropriate community (professional or classroom). We extend this idea of representation systems and, in subsequent sections, use them to define which arguments will be convincing and explanatory.

Following Weber and Alcock (2009) and consistent with Balacheff (2009), we define a representation system (RS) as a structure with permissible configurations and inferential schemes. Permissible configurations are organizations of inscriptions (words, symbols, lines) that represent mathematical objects and relationships between these objects. Permissible

\footnotetext{
${ }^{1}$ Providing such a definition was not necessarily their intent.
} 
configurations include equations, graphs, and some English sentences. Inferential schemes allow the user to infer new mathematical statements from other mathematical statements (where statements are represented as permissible configurations). A personal inferential scheme is an individual's (possibly pragmatic and defeasible) means to infer new statements from permissible configurations. A truth-preserving inferential scheme is an inferential scheme that the individual believes will always yield true statements from true premises. A valid inferential scheme is an inferential scheme that is regarded by the mathematical community as truth-preserving and acceptable within a proof.

To illustrate, consider the combinatorial notion of " $n$ choose $k$," denoted $\left(\begin{array}{l}n \\ k\end{array}\right)$. This notion can be reasoned about in many different RSs, and we exemplify just two in this section (we elaborate a third in Proof $\mathrm{C}$ below). First, we consider an enumerative $R S$, the $\mathrm{RS}$ in which Proof 1 in the beginning of this paper is couched. Here " $n$ choose $k$ " is represented by natural language sentences describing real-life situations in which one chooses $k$ objects from a set of $n$ objects, such as when choosing committees (these words and sentences are the respective characters and permissible configurations of the system). A crucial valid inferential scheme for this RS is that if two different combinatorial expressions can be represented as different ways of counting the same set of objects, then the two combinatorial expressions are equal. Other valid inferential schemes may draw on ideas like the multiplication principle or equivalence.

We can also consider an algebraic $R S$ in which Proof 2 is couched. Here, " $n$ choose $k$ " can be represented by the expression $\frac{n !}{(n-k) ! k !}$, where characters involve symbols and variables, and permissible configurations include algebraic expressions and equations. A crucial valid inferential scheme for this RS is the transitive property for equality of real numbers. Other valid inferential schemes include general algebraic techniques and properties of factorials.

The RS in which an individual chooses to reason may have a significant impact on the types of arguments she can construct. Arguments within one RS may be difficult to replicate in another RS. For instance, we do not see how one could couch Proof 1 in an algebraic RS or Proof 2 in an enumerative RS. However, even if there is a correspondence between two RSs, an inference within one RS may take less cognitive effort than the analogous inference in the other. To illustrate, consider Figure 6 (taken from Sfard, 1991), which represents the same mathematical concept in two different ways. Now imagine trying to justify that the shortest stroll between 12 and 4 is four steps. It seems clear that producing a justification in the second graphical RS is less cognitively demanding than in the first, and will likely be easier to understand.

We refer to personal inferences that are easy for an individual to produce and understand because of her familiarity with an RS as natural inferences. We make three important clarifications. First, what inferences are natural depends on the individual. In Figure 6, viewing $\operatorname{North}(x)$ as moving up a node is intuitive based on our experience reading maps and reasoning about diagrams. For someone without such experience, the visual RS in Figure 6 might not permit natural inferences. Second, with sufficient experience within an RS, some inferences may become natural to an expert, even if they seem mysterious to a layperson. For instance, those with substantial experience in high school algebra will find it natural to simplify expressions by cancelling like expressions from the numerator and denominator, even though some learners might find such simplifications challenging. Third, natural inferences are not necessarily truthpreserving or valid. For instance, concluding that a function is increasing because its graph goes up as it is read from left to right is both defeasible and generally not permissible in a proof (e.g., Weber \& Mejía-Ramos, 2019). 


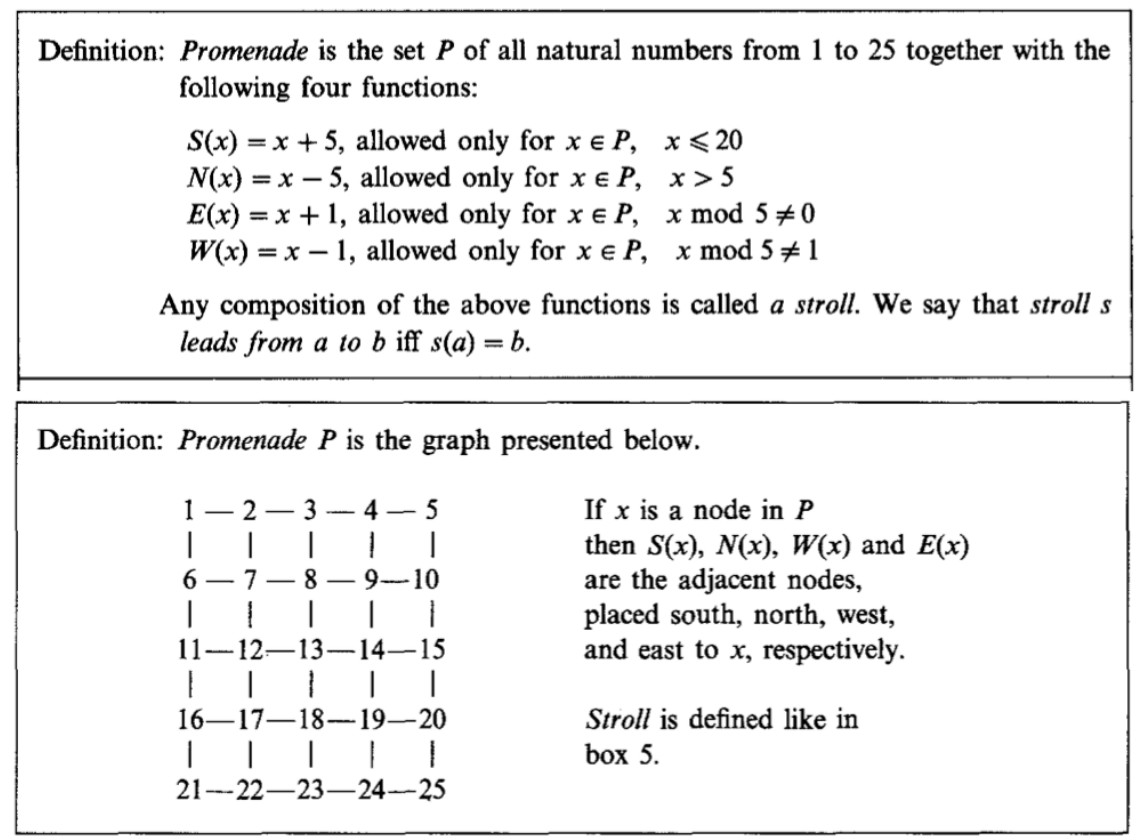

Figure 6. Sfard's (1991) representations of Promenades and Strolls.

To keep our discussion manageable, we will focus on students reasoning in RSs in which there is a shared understanding between the student and the community as to which inferential schemes are permissible. This is an oversimplification in two respects. First, students often are reasoning in RSs where they do not understand the difference between personal and valid inferential schemes (Balacheff, 2009), so with regard to explanatory proofs, we are speaking to relatively mature students. Second, even in professional mathematical communities, there is often ambiguity as to which inferential schemes are valid or require further justification. Generalizing our framework beyond our simplified scope is an interesting project for future research.

\section{Convincing Arguments and Their Relationship to Proof}

We consider a mathematical argument to be convincing to an individual when it has the following characteristics: (i) it begins with axioms, definitions, or statements the individual believes are true, (ii) it employs personal inferential schemes the individual believes are truthpreserving, and (iii) it is couched within, or can be mapped to, an RS the individual finds personally meaningful. By personally meaningful, we mean the individual understands how the permissible configurations in the RS represent the relationship between the mathematical ideas under consideration.

To us, the biggest difference between evaluating whether an argument is convincing or qualifies as a proof is that the former is a personal psychological judgment while the latter is inherently a social judgment. In our view, evaluating whether an argument is a proof requires one to consider whether the argument is appropriate to the community. There is no such requirement in our characterization of whether an argument is convincing.

With regard to (i), Stylianides' (2007) description requires proof begin with arguments that are accepted statements by a community. We presume if a statement is not an axiom or 
definition, the statement would need to have been proven to be accepted. For a convincing argument, the bar is lower. The individual need only believe the statement is true, even if her community has not yet accepted it. For instance, imagine a mathematician who believes that the Riemann Hypothesis is true (as many mathematicians do). She may find an argument showing that statement $\mathrm{S}$ that is implied by the Riemann Hypothesis to be a convincing argument for $\mathrm{S}$ despite being aware that this is not a proof of S, since the Riemann Hypothesis is not a theorem.

With regard to (ii), an individual may believe some inferential schemes are truth-preserving even if she is aware that they are beyond the reach of her classroom community or that the mathematical community would not regard them as valid. For instance, students may gain conviction by generalizing from examples even if they are aware this is impermissible in a proof (e.g., Brown, 2014).

With regard to (iii), an individual may consider an RS as personally meaningful even if she is aware that the mathematical community regards it as inappropriate for proof. For instance, in his Proofs Without Words, Nelsen (1993) indicates he finds picture arguments to be convincing but does not think they qualify as proofs. Similarly, Weber (2010b) describes a student who finds a graphical justification to be "completely convincing" but nonetheless not to qualify as a proof. The student's rationale is: "they don't really allow us to use graphs. And this, I'm basing this whole proof off of a graph which is not, I would say, professional... So, that's what I've been learning in class. We're not allowed to draw any pictures." (p.323). Here a student distinguishes between RSs in which the inferential schemes are personally convincing to him and those which he believes are valid. Finally, while the examples above illustrate how individuals may find an argument convincing while not being a proof, it can also be the case that there are proofs that are not convincing. This can occur, for instance, if there is an inferential scheme that an individual knows the mathematical community accepts as valid but about which the individual has personal doubts. This can occur with students if an argument is couched in an RS that the student does not really understand. This likely occurs rarely in mathematical practice; but this may occur with students, such as when they accept proofs as valid yet nonetheless find them unpersuasive.

\section{Explanatory Arguments and Their Relationship to Conviction and Proof}

We define a mathematical argument as explanatory to an individual with respect to an RS when it has the following characteristics: (i) it begins with axioms, definitions, or statements the individual believes are true, (ii) it employs inferential schemes that are natural, and (iii) it is couched within, or can be mapped to, an RS the individual finds personally valuable.

By personally valuable, we mean a personally meaningful RS that an individual additionally feels would be interesting or appropriate to couch a proof. Individuals' reasons for personally valuing an RS may be idiosyncratic, but we offer two general factors that students or mathematicians may consider. First, some mathematicians prefer RSs that place a proof in its "proper context," meaning that the RS relates to the primary meanings of the concepts involved in the proven statement (Paseau, 2010). For instance, if an individual thought that $\left(\begin{array}{l}n \\ k\end{array}\right)$ really was about choosing $k$-element subsets from an $n$ element set, and felt that the formula $\frac{n !}{(n-k) ! k !}$ was a secondary expression that just happens to algebraically capture the number of ways to choose these subsets, she would likely value the enumerative RS of Proof 1. Alternatively, an individual who felt that $\left(\begin{array}{l}n \\ k\end{array}\right)$ really was an abbreviation for $\frac{n !}{(n-k) ! k !}$, and felt that choosing $k$-element subsets from an $n$ element sets was an interesting interpretation or application of the expression 
abbreviated by $\left(\begin{array}{l}n \\ k\end{array}\right)$, would likely value the algebraic RS of Proof 2. As Paseau (2010) notes, mathematicians may disagree on the 'proper context' for a proof. Second, an RS may be personally valued by an individual if it aligns with that individuals' preferred reasoning style. For instance, an individual who prefers to reason visually will tend to value RSs that represent concepts diagrammatically and allow for perceptual inferences.

As with the relationship between conviction and proof, we again observe that in our framing not every explanation is a proof and not every proof is explanatory. The differences between proof and explanation parallel the differences between proof and conviction laid out earlier evaluating an argument as a proof involves a social judgment and evaluating an argument as explanatory is a psychological judgment. For instance, many individuals such as Nelsen (1993) find some of Nelsen's "proofs without words" to be explanatory (because they rely on natural inferences) but not to qualify as bona fide proofs (because they occur in an inappropriate RS) ${ }^{2}$.

In the mathematics education literature, there is a focus on explanatory proofs - that is, arguments that are both explanatory and proofs. For the remainder of this paper, we will focus on explanatory proofs, contrasting them with convincing proofs that are not explanatory. We highlight four consequences from our definition of explanatory arguments.

We first contend that explanatory proofs will generally be convincing as well. Criterion (i) for conviction and explanation are identical. With regard to (ii), in our conception, a truthpreserving inference need not be natural to an individual. An individual can verify a cumbersome calculation without finding it to be natural (i.e., the calculation may require substantial cognitive effort). Alternatively, as previously noted, a natural inference need not be truth-preserving. However, in general, if an individual finds an inference to be natural within an RS she understands and finds valuable (criteria (ii) and (iii) for explanation) and she believes that mathematicians would find the inference to be valid (a criterion for proof), the individual will typically find the inference to be truth-preserving (criterion (ii) for conviction). Finally, criterion (iii) for explanation implies criterion (iii) for conviction. That is, if an RS is personally valuable, it will be personally meaningful.

Second, this definition views explanations as explaining how rather than why. This is consistent with the way mathematicians use the word "explain" in their writing. Based on a corpus analysis of a large number of mathematical articles, Mejia-Ramos and Inglis (2017) found that mathematicians were far more likely to use the phrase "explain how" in their text than "explain why." Note that by emphasizing explaining how we are not suggesting that an explanatory proof merely explains how to carry out steps in a procedure; justification is central to our understanding of an explanatory proof, and we propose that an explanatory proof outlines a method for how a statement might be justified. That is, by explaining how a statement can be justified, the proof makes explicit why that statement is true.

Third, proofs are not globally explanatory, but explanatory with respect to a given RS. What is being explained is how to justify a claim within a desirable RS or with a particular type of reasoning. For instance, Proof 1 explains how to prove an identity enumeratively, and Proof 2 explains how to prove it algebraically. We explore this further in the subsequent section.

\footnotetext{
${ }^{2}$ Balacheff (2009) also defined explanation as a psychological construct, proof as a social construct, and both characterized in terms of representation systems. However, at least in terms of student-generated proofs, he viewed a proof as a socially accepted explanation (i.e., all proofs are explanations). Our characterization says a studentgenerated proof might not be explanatory if it was generated with non-natural inferences or in an RS that a student did not personally value.
} 
Fourth, this definition is clearly student-centered. With respect to (ii), recall we defined natural inferences as inferences that are easy for an individual to produce and understand because of her familiarity with an RS. Whether an inferential scheme is natural is partially dependent upon the individual's experience working within that RS. Further, with respect to (iii), the types of RSs that are considered valuable can vary between individuals. Thus, we reiterate that RSs (and therefore the extent to which a certain proof might be considered explanatory) are closely related to an individual's prior knowledge and background experience.

\section{Clarifying Aspects of Explanatory Proof through a Discussion of Mathematical Examples in a Combinatorial Context}

\section{Insights from Proofs of Binomial Identities}

To elaborate our definitions of RSs and explanatory proof, we consider examples from combinatorics involving binomial identities. In our experience, this topic naturally lends itself to moving between RSs. Indeed, as we will describe, it is commonplace to use a secondary RS (perhaps enumerative) to prove a relationship in a given RS (perhaps algebraic). To appreciate the upcoming discussion, take a moment to consider the following identity. For all integers $0 \leq k$ $\leq n$, the binomial coefficients satisfy:

$$
\left(\begin{array}{l}
n \\
k
\end{array}\right)=\left(\begin{array}{c}
n \\
n-k
\end{array}\right) .
$$

When you see equation (2), how do you interpret the claim being made? Is it a claim about two coefficients in a polynomial? Is it an assertion about two types of subsets of an $n$-element set? Is it describing a property of a recursively defined triangular array (Pascal's triangle)? Is it saying several of these? Something else? Depending on how you answer this question, you may have different expectations of what constitutes a "proof that explains," and you may find different proofs more or less explanatory, or even more or less convincing.

In this section we provide proofs of claim (2) in three different representation systems. We offer proofs in RSs that emphasize enumeration (Proof A), rules of algebra (Proof B), and the binomial theorem (Proof C), acknowledging that we could also explore additional RSs of this same expression (such as induction, block-walking, Taylor's theorem, etc.).

Proof A: An explanatory proof in an enumerative RS. In an enumerative proof, we argue that the two sides of the identity represent two different counting processes (e.g., Lockwood, 2013) that either a) count the same set of outcomes (a direct combinatorial proof) or b) count two different sets of outcomes between which there is a bijection (a bijective combinatorial proof). Valid inferential schemes include enumerative arguments that draw on fundamental combinatorial principles and the fact that sets have a unique cardinality. For simplicity, we provide one example of a direct combinatorial proof.

We show that both sides of the identity count the following set: the set of $k$-member committees of a group of $n$ people. That is, we interpret equation (2) as a statement that relates different expressions counting subsets of $n$-element sets. The left-hand side counts the number of $k$-member committees by selecting $k$ members from the $n$ people that should be included in the committee. The right-hand side counts the number of $k$-member committees by enumerating their complements - by selecting the $n-k$ group members from $n$ people who will not serve on the committee. Because both sides of the identity count the same quantity (the number of $k$-member committees), the expressions must be numerically equal. This proof explains how we justify the 
combinatorial identity in terms of counting subsets; in this way, we gain insight into why the statement is true.

Proof B: An explanatory proof in an algebraic RS. We can accept the enumerative justification above as being both convincing and a proof, yet we can still wonder about how the combinatorial identity can be justified algebraically. In an algebraic RS, equation (2) can be interpreted as a statement about factorial expressions involving (nonnegative) integers, and valid rules of inference include properties of integers and algebraic rules.

Substituting the algebraic interpretation of binomial coefficients $\left(\begin{array}{l}n \\ k\end{array}\right)=\frac{n !}{(n-k) ! k !}$ into (2) and applying rules of algebra yields the following proof:

$$
\left(\begin{array}{l}
n \\
k
\end{array}\right)=\frac{n !}{(n-k) ! k !}=\frac{n !}{k !(n-k) !}=\frac{n !}{(n-(n-k)) !(n-k) !}=\left(\begin{array}{c}
n \\
n-k
\end{array}\right) .
$$

Since we can use rules of algebra to manipulate and transform one expression into the other, both sides of (2) are algebraically equivalent, and the statement follows. This algebraic proof explains something different than the enumerative proof - how we justify the identity in terms of algebraic rules and inferences. By detailing how the identity can be justified algebraically, it proves directly why the algebraic claim is true. These first two proofs, A and B, are similar to the examples of Proofs 1 and 2 from the introduction.

Proof C: An explanatory proof in a binomial theorem RS. In another RS, we could perceive binomial coefficients $\left(\begin{array}{l}n \\ k\end{array}\right)$ in terms of their role in the binomial theorem. The theorem states $^{3}$ that for all nonnegative integers $a, b$, and $n$,

$$
(a+b)^{n}=\sum_{k=0}^{n}\left(\begin{array}{l}
n \\
k
\end{array}\right) a^{k} b^{n-k} .
$$

Thus the interpretation of $\left(\begin{array}{l}n \\ k\end{array}\right)$ is as the coefficient of the term $a^{k} b^{n-k}$ in the expansion of the expression $(a+b)^{n}$. In this RS, valid rules of inference involve rules of algebraic expansion and the equality of corresponding coefficients.

To justify (2) in this sense, we consider the following:

Proof: Consider the expansion of $(b+a)^{n}$. Here, the term $\left(\begin{array}{c}n \\ n-k\end{array}\right)$ represents the coefficient of $b^{n-k} a^{n-(n-k)}=b^{n-k} a^{k}$. By commutativity of addition, $(a+b)^{n}=(b+a)^{n}$, and so our two expansions should agree. By commutativity of multiplication, $a^{k} b^{n-k}=b^{n-k} a^{k}$, and so these coefficients should agree. Therefore, because $\left(\begin{array}{l}n \\ k\end{array}\right)$ is the coefficient of $a^{k} b^{n-k}$ in the expansion of $(a+b)^{n}$, and because $\left(\begin{array}{c}n \\ n-k\end{array}\right)$ is the coefficient of $b^{n-k} a^{k}$ in the expansion of $(b+a)^{n}$, and because the expansions and their terms must agree, we conclude that equation (2) holds.

Again, this proof explains how (2) can be derived within the context of the binomial theorem, and in doing so, it makes explicit why the binomial coefficients in (2) must be equal. Like Proof $\mathrm{B}$, this proof also relies on algebra, but the proofs are different in the ways they appeal to specific rules of inference appropriate to the respective RSs. Here the initial step involves interpreting the two sides of (2) not as an algebraic expression of $\frac{n !}{(n-k) ! k !}$, but rather as specific coefficients of two binomial expansions.

\section{Reflections on the Algebraic and Enumerative Proofs - What Is Being Explained?}

\footnotetext{
${ }^{3}$ The theorem extends to other, non-integer values, but we focus on this version.
} 
We take these three proofs as reference points for continuing our discussion of proofs that explain and proofs that only convince. A common way to view these proofs would be to say that the algebraic Proof B only convinces, while the enumerative Proof A is somehow more explanatory (and perhaps Proof $\mathrm{C}$ is somewhere in between). One could argue that the enumerative proof involves some evocative real-world activity (choosing committees) while the algebraic proof involves merely symbol-pushing. We understand the appeal of this perspective, and as combinatorialists we find the enumerative proofs to be particularly satisfying. However, we argue that there is a deeper story to tell, and each of the above proofs can be considered to be explanatory and/or convincing depending on which RS a certain individual might adopt.

We contend that the question What is the proof explaining? is not a simple inquiry, but it is made somewhat clearer when viewed in the context of RSs. For example, we would argue that the enumerative Proof A is explanatory in the enumerative RS because it demonstrates how (2) can be justified in terms of counting subsets (in particular, committees). However, this enumerative proof is not explanatory in an algebraic RS or in an RS focused on the binomial theorem. That is, even if one fully understands the enumerative Proof A, there still remains the mystery of why the result follows if we interpret $\left(\begin{array}{l}n \\ k\end{array}\right)$ as $\frac{n !}{(n-k) ! k !}$ or as a coefficient of a binomial expansion. Conversely, the algebraic Proof B does not provide explanation for how the identity might be justified in an enumerative domain; however, Proof B is explanatory in the RS of algebra, interpreting $\left(\begin{array}{l}n \\ k\end{array}\right)$ as a particular quotient of factorial expressions.

In short, what constitutes an explanation is relative to the RS used to interpret the claim being made. How does this relate to convincing? We contend that a proof of a claim in a given RS may be convincing to an individual but not explanatory for them, particularly if they interpret the claim using another RS. In our examples of multiple proofs of the identity (2), we suggest that the algebraic proof may be convincing to an individual who reads (2) as a claim in the enumerative RS, even if it is not explanatory to them (it does not explain the enumeration). This is especially true if the individual acknowledges the validity of the identity $\left(\begin{array}{l}n \\ k\end{array}\right)=\frac{n !}{(n-k) ! k !}$, which can function as a translation device between the enumerative and algebraic RSs. Similarly, the enumerative proof may convince someone that the algebraic claim must be true, even if the enumerative proof offers no insight into what specific algebraic steps could be used to verify this fact in an algebraic RS.

We commonly use such relationships between RSs to prove results in combinatorics. To emphasize this point, consider the following identity

$$
\sum_{k=0}^{n}\left(\begin{array}{l}
n \\
k
\end{array}\right)=2^{n}
$$

This identity is quite natural to prove enumeratively. Both sides count the total number of subsets of any size from a set of $n$ elements. The left-hand side counts this by summing the numbers of $k$-element subsets for all possible values of $k$ from 0 to $n$. The right-hand side counts this by considering, for each of the $n$ elements in the set, whether or not to include it as an element of a subset. Identity (4) is also straightforward to prove using the binomial theorem, substituting the value 1 for both $a$ and $b$ in (3). However, it is not immediately apparent why the corresponding algebraic fact about sums of quotients of factorial expressions should hold (just try it). Here, then, an enumerative proof may convince us of the ultimate correctness of the algebraic claim, and it may give us confidence that an algebraic derivation exists, but it does not itself explicitly reveal or fully explain the conjectured algebraic claim. In other words, if all we needed was to be convinced that this identity holds, it would suffice to use an enumerative argument or the 
binomial theorem to prove the result, rather than an algebraic argument. But if we sought to understand why such a miraculous algebraic claim holds algebraically, we might remain just as mystified after these proofs as we were before.

More commonly in combinatorics research, we proceed in the other direction - using algebra to convince us of identities that are difficult to prove enumeratively. For example, generating functions offer a well-established technique of translating combinatorial questions into algebraic settings, using algebra to derive results, then translating back into the combinatorial context. A proof by generating functions is explanatory in an algebraic RS, demonstrating clearly how algebraic manipulations establish the relationship, but it does not necessarily explain how the relationship may be justified enumeratively.

The fact that there are different RSs in which proofs may convince or explain is a wonderful aspect of mathematics, affording opportunities to develop convincing proofs even if the derivation of a claim in a preferred RS is particularly difficult. Our point, then, is that it is overly simplistic to characterize a proof as being objectively convincing or explanatory without further specifying what precisely is being explained and to whom, and in particular, in what RS the statement to prove is being interpreted.

\section{Relation to Previous Insights about Explanatory Proof}

Earlier, we summarized five insights about explanatory proofs from the mathematics education literature for which a good characterization of explanatory proof should account. Here we argue that our characterization meets these challenges.

Explanatory proofs should be individual-dependent. Whether a proof is explanatory to an individual depends critically on which RSs that individual understands and personally values. As different students have different experiences, interests, and reasoning preferences, their relationship to specific RSs will also be different. A student's tendency to value algebra over enumeration may stem from an overall comfort level with algebra and a feeling of inadequacy or inexperience in being able to solve counting problems (indeed, student difficulties with combinatorics are well documented, e.g., Batanero, et al., 1997). For such students, it is understandable that they would value algebra over counting, as an algebraic RS would likely allow them to feel more comfortable and confident in their proof. Similarly, a mathematician may value an enumerative proof because it offers insight about an enumeration technique that they value, even if they are just as comfortable and confident about algebra.

There is some uniformity about which proofs are explanatory. In the next two paragraphs, we describe two areas of such uniformity, the tendency of visual arguments to be explanatory and the reasons why complicated algebraic arguments tend not to be.

Proofs that rely on visual reasoning tend to be explanatory. As Bartlo (2013) observes, mathematics educators tend to offer visual proofs as explanatory proofs. These proofs usually involve inferential schemes based on our intuitions about two-dimensional Euclidean space (e.g., Weber \& Mejia-Ramos, 2019) or on geometry facts learned at a young age. For instance, Hanna's (1990) explanatory proof in Figure 2 relies on basic geometric intuition and facts. For most students with extensive mathematical training, the geometric RS in which Hanna is working will be understood well and the inferential schemes relying on two-dimensional Euclidean space will be natural. This is one reason why justifications couched within a visual RS tend to be viewed as explanatory.

Proofs that rely on complex algebraic manipulations tend not to be explanatory. Bartlo (2013) further observes that the most frequent exemplars of non-explanatory proofs rely on 
complicated algebra. We suggest that the fact that the algebraic manipulations are complicated implies that the algebraic inferences being made are framed as requiring substantial cognitive effort and not being natural. Standard template-based proofs by induction (c.f. Harel, 2001), such as the proof in Figure 4 that Hanna (1990) highlights as non-explanatory, offer an interesting special case of this. Many of the algebraic inferences are truth preserving upon reflection, but they are not natural inferences that can easily be seen by dint of the algebraic RS. Further, the

overarching structure of the inductive step involves deducing $S(k+1)=\frac{(n+1)(n+2)}{2}$ from the inductive hypothesis. Again, an individual who understood proof by induction could verify that this (along with the base case) was sufficient to prove the theorem, but this is not obvious on the surface, and is often not how we think about "what the theorem statement is really about". For instance, unlike Hanna's (1990) explanatory proof presented in Figure 5, the proof does not represent the summation of $n$ numbers as the accumulation of $n$ quantities. (However, Harel (2001) and Stylianides et al. (2016) suggest that proofs by induction can be explanatory if they are manifestations of students' natural recursive reasoning). In addition, it may be the case that complex algebraic proofs do not typically explain what an individual wants to be explained. That is, as in the case of combinatorial identities, often algebraic expressions serve as a proxy for different mathematical phenomena or objects that an individual values, and the algebra in and of itself is not what an individual seeks to justify or explain about a statement.

Students are more likely to find their own proofs, which are the result of their own proving process, to be explanatory. As Stylianides et al. (2016) and Nunokawa (2009) observe, under some conditions, students are likely to view a proof in which the students' final proof product is based on their initial exploration to be explanatory. We believe this insight is a consequence of our characterization of explanation. Students will tend to explore the veracity of conjectures in RSs that they understand well and personally value. Hence, when students decide that a conjecture is true, they do so based on an explanatory argument that they generated. If a cognitive unity (Garuti et al., 1998) between this argument in their proof is achieved, then the individual will perceive a mapping between their explanatory argument and the proof that they produced. This satisfies our condition for a proof to be explanatory.

\section{Conclusion}

Our goals in this paper were threefold: to propose a theory of explanatory proofs for mathematics education, framed in terms of the representation systems in which proofs are developed, written, and understood; to use combinatorics as a domain in which to illustrate our proposed theory; and to leverage our theory to explain audience-dependent and audienceinvariant aspects of explanatory proof. Wanting to move beyond simply labeling proofs as explanatory, we hope to have contributed to a more nuanced perspective on proofs that explain.

The notion of RSs in proof allows us to reframe how we think about students' proving activity. The idea that students may work from different RSs gives a lens through which to consider student activity in proof-based classes, perhaps giving students more credit than simply dismissing their activity as meaningless and purely syntactic. For example, when a student tends toward algebra when trying to prove a binomial identity, it is easy to assume they are engaging superficially, not sufficiently attempting to understand the situation conceptually. But such a student may be viewing the statement to be proven through an algebraic RS, which may represent the primary meaning of the claim to them. By clarifying how arguments might be construed as explanatory or convincing to an individual (and by suggesting that arguments may be explanatory in a variety of different ways), we allow for potentially broader views of how 
individuals make sense of and use mathematical concepts, ideas, or perspectives. Thus, this perspective on proofs that explain may give agency to the prover. 


\section{Acknowledgments}

We thank the anonymous reviewers for helpful comments. 


\section{References}

Batanero, C., Navarro-Pelayo, V., \& Godino, J. (1997). Effect of the implicit combinatorial model on combinatorial reasoning in secondary school pupils. Educational Studies in Mathematics, 32, 181-199.

Balacheff, N. (2009). Bridging knowing and proving in mathematics: A didactical perspective. In Pulte, H., Hanna, G., \& Jahnke, H. J. (Eds.). (2009). Explanation and proof in mathematics: philosophical and educational perspectives. New York: Springer.

Bartlo, J. R. (2013). Why ask why: an exploration of the role of proof in the mathematics classroom. (Unpublished doctoral dissertation). Portland State University. Portland, OR.

Brown, S. A. (2014). On skepticism and its role in the development of proof in the classroom. Educational Studies in Mathematics, 86(3), 311-335.

De Villiers, M. (1999). The role and function of proof. In M. de Villiers (Ed.), Rethinking proof with the Geometer's Sketchpad (pp. 3-10). Key Curriculum Press.

Fischbein, E. (1987). Intuition in science and mathematics: an educational approach (Vol. 5). Springer: Dordrecht.

Garuti, R., Boero, P., \& Lemut, E. (1998). Cognitive unity of theorems and difficulty of proof. In A. Olivier \& K. Newstead (Eds.), Proceedings of the 22nd Conference of the International Group for the Psychology of Mathematics Education (Vol. 2, pp. 345-352). Stellenbosch, South Africa.

Hanna, G. (1990). Some pedagogical aspects of proof. Interchange, 21(1), 6-13.

Harel, G., \& Sowder, L. (1998). Students' proof schemes. In E. Dubinsky, A. Schoenfeld, \& J. Kaput (Eds.), Research on Collegiate Mathematics Education (Vol. III) (pp. 234-283). Providence, RI: American Mathematical Society.

Harel, G. (2001). The development of mathematical induction as a proof scheme: A Model for DNR-based instruction. In S. Campbell \& R. Zaskis (Eds.), Learning and teaching number theory (pp. 185-212). Norwood, NJ: Ablex.

Hersh, R. (1993). Proving is convincing and explaining. Educational Studies in Mathematics, 24(4), 389-399.

Lange, M. (2009). Why proofs by mathematical induction are generally not explanatory. Analysis, 69(2), 203-211.

Lockwood, E. (2013). A model of students' combinatorial thinking. Journal of Mathematical Behavior, 32, 251-265. doi:10.1016/j.jmathb.2013.02.008

Mejia-Ramos, J.P. \& Inglis, M. (2017). 'Explanatory' talk in mathematics research papers. In (Eds.) A. Weinberg, C. Rasmussen, J. Rabin, M. Wawro, and S. Brown Proceedings of the $20^{\text {th }}$ Conference for Research in Undergraduate Mathematics Education (pp. 373382). San Diego, California.

Nelsen, R. (1993). Proofs without words: exercises in visual thinking. Washington, DC: MAA.

Nunokawa, K. (2009). Proof, mathematical problem-solving, and explanation in mathematical teaching. In Pulte, H., Hanna, G., \& Jahnke, H. J. (Eds.). (2009). Explanation and proof in mathematics: philosophical and educational perspectives. New York: Springer.

Paseau, A. (2010). Proofs of the compactness theorem. History and Philosophy of Logic, 31(1), 73-98.

Pulte, H., Hanna, G., \& Jahnke, H. J. (Eds.). (2009). Explanation and proof in mathematics: philosophical and educational perspectives. New York: Springer. 
Raman, M. (2003). Key ideas: What are they and how can they help us understand how people view proof? Educational Studies in Mathematics, 52(3), 319-325.

Sfard, A. (1991). On the dual nature of mathematical conceptions: Reflections on processes and objects as different sides of the same coin. Educational Studies in Mathematics, 22, 1-36.

Steiner, M. (1978). Mathematical explanation. Philosophical Studies, 34, 135-151.

Stylianides, A. J. (2007). Proof and proving in school mathematics. Journal for Research in Mathematics Education, 38(3), 289-321.

Stylianides, G. J., Sandefur, J., \& Watson, A. (2016). Conditions for proving by mathematical induction to be explanatory. Journal of Mathematical Behavior, 43, 20-34.

Weber, K. (2010a). Proofs that develop insight: proofs that reconceive mathematical domains and proofs that introduce new methods. For the Learning of Mathematics, 30(1), 32-37.

Weber, K. (2010b). Mathematics majors' perceptions of conviction, validity, and proof. Mathematical Thinking and Learning, 12, 306-336.

Weber, K. \& Alcock, L. (2004). Semantic and syntactic proof productions. Educational Studies in Mathematics, 56, 209-234.

Weber, K. \& Alcock, L. (2009). Semantic and syntactic reasoning and proving in advanced mathematics classrooms. Invited chapter on research on proof at the undergraduate level for M. Blanton, D. Stylinaiou, and E. Knuth (Eds.), The teaching and learning of proof across the K-16 curriculum (pp. 323-338). New York: Routledge.

Weber, K. \& Mejia-Ramos, J.P. (2019). An empirical study on the admissibility of graphical inferences in mathematical proofs. In A. Aberdein \& M. Inglis (Ed.) Advances in experimental philosophy of logic and mathematics (pp. 123-144). London: Bloomsbury. 